\title{
Transplantation of Embryonic Fibroblasts Treated with Platelet-Rich Plasma Induces Osteogenesis in SAMP8 Mice Monitored by Molecular Imaging
}

\author{
Wen-Cheng Lo ${ }^{1}$, Jeng-Fong Chiou ${ }^{2,3}$, Juri G. Gelovani ${ }^{4}$, Mei-Leng Cheong ${ }^{5}$, Chi-Ming Lee ${ }^{6}$, Hen-Yu Liu ${ }^{7}$, \\ Chih-Hsiung $\mathrm{Wu}^{8}$, Ming-Fu Wang ${ }^{9}$, Che-Tong Lin $^{10}$, and Win-Ping Deng ${ }^{11}$ \\ ${ }^{I}$ Graduate Institute of Clinical Medicine, Taipei Medical University, Taipei, Taiwan; ${ }^{2}$ Cancer Center and \\ Department of Radiation Oncology, Taipei Medical University Hospital, Taipei, Taiwan; ${ }^{3}$ Institute of Medical Sciences, National \\ Defense Medical Center, Taipei, Taiwan; ${ }^{4}$ Experimental Diagnostic Imaging, M.D. Anderson Cancer Center, Houston, Texas; \\ ${ }^{5}$ Department of Obstetrics and Gynecology, Cathay General Hospital, Taipei, Taiwan; ${ }^{6}$ Department of Diagnostic Radiology, \\ Taipei Medical University Hospital, Taipei, Taiwan; ${ }^{7}$ Graduate Institute of Medical Sciences, Taipei Medical University, Taipei, \\ Taiwan; ${ }^{8}$ Division of General Surgery, Department of Surgery and Cancer Center, Taipei Medical University Hospital, Taipei, \\ Taiwan; ${ }^{9}$ Department of Food and Nutrition, Providence University, Taichung, Taiwan; ${ }^{10}$ Department of Prosthetic Dentistry, \\ School of Dentistry, College of Oral Medicine, Taipei Medical University, Taipei, Taiwan; and ${ }^{11}$ Graduate Institute of \\ Biomedical Materials and Engineering, Taipei Medical University, Taipei, Taiwan
}

The aim of this study was to develop a cell-based boneregeneration approach evaluated by molecular imaging and immunohistochemistry. Methods: Genetically modified NIH3T3 embryonic fibroblasts carrying enhanced green fluorescent protein (NIH3T3-G) were predifferentiated into osteoblastlike cells using platelet-rich plasma (PRP) medium, followed by intraosseous transplantation into ovariectomized senescenceaccelerated mouse prone substrain 8 (OVX-SAMP8 mice). Results: PRP-conditioned NIH3T3-G (PRP/NIH3T3-G) engraftment prevented the development of osteoporosis. Molecular imaging and immunohistochemistry demonstrated the migration of NIH3T3-G cells from the implantation site throughout the skeleton. In situ analyses revealed coexpression of osteopontin and green fluorescent protein in the newly formed bone tissue, demonstrating that the transplant restored the bone trabecular architecture and mineral density in treated OVX-SAMP8 mice. Interestingly, the life span of OVX-SAMP8 mice receiving PRP/ NIH3T3-G transplantation was significantly prolonged and similar to that of the congenic senescence-resistant strain of mice. Conclusion: This unique and yet simple approach could potentially be applied to the treatment of senile postmenopausal osteoporosis and perhaps inborn genetic syndromes associated with accelerated aging, such as Hutchinson-Gilford progeria syndrome, and for the prolongation of life expectancy in general.

Key Words: bone; molecular biology; molecular imaging; osteogenesis; platelet-rich plasma; transplantation; senescenceaccelerated mice P8

J Nucl Med 2009; 50:765-773

DOI: 10.2967/jnumed.108.057372

Received Aug. 25, 2008; revision accepted Nov. 6, 2008

For correspondence or reprints contact: Win-Ping Deng, Graduate Institute of Biomedical Materials and Engineering, Taipei Medical

University, 250 Wu-Hsing St., Taipei, 110, Taiwan.

E-mail: wpdeng@tmu.edu.tw

COPYRIGHT @ 2009 by the Society of Nuclear Medicine, Inc.
O steoporosis is a disorder characterized by a significant reduction in total bone mass and compromised bone tissue quality, resulting in increased risks for fractures, particularly of the spine, hips, and limbs. Osteoporosis is classified into type I (postmenopausal osteoporosis, which occurs in women after menopause) and type II (senile osteoporosis, which affects elderly men and women). The underlying mechanism of age-related bone loss involves osteoblast dysfunction coupled with various systemic abnormalities (1). It is estimated that more than 200 million people worldwide have osteoporosis and experience associated morbidity, mortality, and decreased quality of life. The prevalence of osteoporosis continues to increase with the aging population globally (2).

Current therapies for osteoporosis are directed at the prevention of excessive bone loss. For instance, bisphosphonates (alendronate, risedronate, and ibandronate) are effective as antiresorptive agents (3). These agents inhibit farnesyl diphosphate synthase, a key enzyme in the mevalonate pathway, to induce apoptosis in osteoclasts (4). A newly available treatment for osteoporosis involves parathyroid hormone-derived peptides. These peptides stimulate bone generation to reinforce bone trabecular microarchitecture caused by estrogen deprivation and reduce the vulnerability to bone fractures (5). Although these therapies are effective to some extent, they are not focused on the major underlying cause in the pathogenesis of senile osteoporosis, namely, the loss of functional osteoblasts during aging (6-9). Recently, cell-based therapies have been vigorously pursued in the hope of mending disorders 
involved in dysfunctional or damaged tissues. Osteoporosis studies have demonstrated that genetically modified dermal fibroblasts overexpressing osteoinductive genes such as Runx2/Cbfa1 (10) and LMP-1 (11) induce osteoblastic differentiation, mineralization, and bone formation to repair bone defects in animals. These previous studies support the use of nonosteogenic fibroblasts as an attractive alternative cell source, because fibroblasts are easily harvested from autologous donors, expanded, and genetically engineered in vitro.

This study investigates the potential use of fibroblasts in combination with platelet-rich plasma (PRP) to achieve bone regeneration in osteoporotic mice. To test this hypothesis, we used a senescence-accelerated mouse (SAM) model representing a pathophysiologic scenario of senile osteoporosis (12). The SAM system was established through phenotypic inbreeding from a common genetic pool of the AKR/J mouse strain. The SAM strain mimics the aging process more accurately than do other genetically altered strains of mice because the SAM phenotype develops from mutations in several genes rather than from a single gene. SAM prone substrain 8 (SAMP8 mice) exhibit many age-related traits, such as learning and memory deficits, anxiety, impaired immune system, and age-dependent amyloid $\beta$-peptide deposition $(13,14)$. Both postmenopausal and senile osteoporoses in SAMP8 mice can be enhanced by ovariectomy, as established by our laboratory.

NIH3T3 embryonic fibroblasts were used for osteoinductive cell transplantation and served as tracers for this study. In addition to the aforementioned advantages of using fibroblasts as a cell model, NIH3T3 fibroblasts have been shown to adopt an osteoblastlike phenotype and exhibit osteogenic activity on appropriate growth factor and cytokine stimulation $(15,16)$. We have genetically engineered the NIH3T3 cells to express the enhanced green fluorescent protein (GFP) reporter gene (NIH3T3-G cells), to facilitate fluorescence imaging of the location, migration, and persistence on transplantation. To stimulate proliferation and differentiation of NIH3T3-G cells into osteoblastlike cells before transplantation, we used PRP medium, which has been shown to induce proliferation of fibroblasts $(17,18)$ and stimulate osteogenesis of rat bone marrow stromal cells $(17,19,20)$.

Here, we demonstrate that transplantation of PRPtreated NIH3T3-G (PRP/NIH3T3-G) cells into ovariectomized SAMP8 mice (OVX-SAMP8 mice) induced bone regeneration and significantly reversed osteoporosis not only at the implantation site but also in other regions of the skeleton. In addition, we have observed an intriguing phenomenon of a marked prolongation of life span in PRP/NIH3T3-G-treated OVX-SAMP8 mice, compared with the vehicle-treated controls. Although additional evidence is required to substantiate the latter finding, this observation could have significant implications in the field of gerontology.

\section{MATERIALS AND METHODS}

\section{Cell Line and Culture}

NIH3T3 embryonic fibroblasts (American Type Culture Collection [ATCC] no. CRL-1658) were purchased from Bioresource Collection and Research Centre and maintained under conditions described by the ATCC. We then genetically modified NIH3T3 cells to stably express the enhanced GFP reporter gene using the Lipofectamine 2000 system (Invitrogen) (NIH3T3-G cells). NIH3T3-G cells were expanded and then enriched $(>95 \%)$ by the fluorescence-activated cell sorter method.

\section{PRP Isolation and Medium Preparation}

Human PRP was prepared and stored at $-20^{\circ} \mathrm{C}$ as previously described (17). Briefly, human whole blood was purchased from Taipei Blood Center and processed using a blood cell-separation system (MCS; Haemonetics Corp.). Bovine thrombin (100 IU bovine thrombin/150 $\mathrm{mL}$ PRP) was then added to the solution to remove aggregated fibrin and centrifuged for $6 \mathrm{~min}(3,000$ revolutions per minute at room temperature). To confirm the consistency of PRP for in vitro use and to determine the most appropriate concentration for the study, transforming growth factor$\beta 1$ (TGF- $\beta 1$ ) was quantitatively analyzed using an enzyme-linked immunosorbent assay kit (Quantikine; R\&D Diagnostics) and used as the core ingredient and a concentration calibrator for PRP measurement (21). The results indicated that a $750 \mathrm{pg} / \mathrm{mL}$ concentration of TGF- $\beta 1$ in PRP was optimal for cell proliferation (19). PRP (calibrated by TFG- $\beta 1$ concentration) was then dissolved in $1 \%$ calf serum (CS) containing Dulbecco's modified Eagle's medium (for basal cell maintenance) and filtered through a $0.22-\mu \mathrm{m}$-pore filter. PRP-containing medium was changed every $2 \mathrm{~d}$ for the proliferation assays, to detect the optimal PRP concentration.

\section{3-(4,5-Dimethylthiazol-2-yl)-2,5-Diphenyltetrazolium Bromide (MTT) Assay}

Cell proliferation was determined by MTT assay (Roche). NIH3T3-G cells were seeded into 96-well plates at a density of $2 \times 10^{3}$ cells $/ \mathrm{mL}$ and treated with PRP in $1 \% \mathrm{CS}$ medium; the control cells were cultured in 1\% CS containing Dulbecco's modified Eagle's medium, without PRP. MTT reagent was added into each well on days 1, 3, 5, and 7 of cell growth in culture. The optical density values were analyzed $4 \mathrm{~h}$ after the MTT reaction using Multiskan PC (Thermo Lab), and cell survival curves were then plotted against time in culture.

\section{Isolation and Culture of Bone Marrow Cells (BMCs)}

Cultured BMCs were obtained from the flushing of the freshly resected femurs and tibia from 4-mo-old SAMP8 mice. The femurs and tibia were cut into pieces and cultured in maintenance medium ( $\alpha$-minimum essential medium, $10 \%$ fetal bovine serum, and $1 \%$ prostate-specific antigen) for $1 \mathrm{wk}$. The nonadherent cells and bone pieces were removed, then washed extensively with phosphate-buffered saline. The adherent cells, BMCs, were collected by trypsin and ethylenediaminetetraacetic acid treatment (Sigma).

\section{Assessment of Osteogenic Differentiation of NIH3T3-G Cells in Vitro}

For the histochemical semiquantitative demonstration of alkaline phosphastase (ALP) activity in cells undergoing osteogenic differentiation, 3 different groups of cells $(\mathrm{NIH} 3 \mathrm{~T} 3-\mathrm{G}$ alone $[1 \times$ $10^{5}$ cells], BMCs alone $\left[1 \times 10^{5}\right.$ cells], and NIH3T3-G/BMC 
monolayer coculture $\left[5 \times 10^{4}\right.$ cells for each population]) were cultured in 6-well dishes either with PRP-enriched medium or with PRP-free medium for $7 \mathrm{~d}$. Cytoplasmic ALP activity in NIH3T3-G cells was determined using a Leukocyte Alkaline Phosphatase kit (Sigma), according to the protocol provided by the vendor. The images were acquired using an inverted microscope (IX71; Olympus) supported by DP controller software (version 2.11.183; Olympus). For the detection of bone nodules by von Kossa staining, NIH3T3-G cells $\left(5 \times 10^{5}\right.$ cells $)$ were seeded into the 10-cm dish and cultured in PRP-free (NIH3T3-G) or PRP-enriched medium (PRP/NIH3T3-G) over the period of $21 \mathrm{~d}$. The staining procedure was performed as previously described (22).

\section{Experimental Animals and Ovariectomy Model}

The animal experiment protocol was approved by the Institutional Animal Care and Use Committee of Taipei Medical University. The average life span of SAMP8 mice and the senescence-accelerated mouse resistant-1 strain (SAMR1 mice) was established by Takeda et al. (12) to be about 12 and $16.6 \mathrm{mo}$, respectively. The SAMP8 mice were ovariectomized at 4 mo after birth (OVX-SAMP8) to induce rapid osteoporosis. Briefly, the ovariectomy was performed under anesthesia; the skin and muscle of the abdomen were incised to expose the gonads. Both ovaries were then removed by scissors, and bleeding was stopped. The muscle and overlying skin were sutured back, and the animals were allowed to recover for $2 \mathrm{wk}$. An additional group of mice $(n=6)$ underwent a sham ovariectomy, in which the same surgical procedure was performed as in the ovariectomized animals, except that the ovaries were not removed.

\section{Mini-Bone Marrow Transplantation}

Various treatment regimens, including the sham vehicle only, NIH3T3-G cells only, PRP only, and PRP-treated NIH3T3-G cells (PRP/NIH3T3-G), were transplanted into OVX-SAMP8 animals by direct injection of cells into the bone marrow cavity. In brief, hair from the right hind limb was removed and a 26-gauge needle was inserted into the joint surface of the tibia through the patellar tendon, followed by injection of cells $\left(10^{6}\right.$ cells $\left./ 10 \mu \mathrm{L}\right)$ with a microsyringe ( $50 \mu \mathrm{L}$; Hamilton Co.) into the bone marrow cavity. After transplantation, the animals were allowed to recover before further examination.

\section{In Vivo Fluorescence Imaging and Analysis}

Mice were sacrificed by cervical dislocation to obtain hind limbs for in vivo fluorescence imaging. The hair and muscles of the limbs were removed before imaging to avoid autofluorescence. The transplanted NIH3T3-G cells were detected with a chargecoupled device camera (IVIS 200; Xenogen).

\section{RNA Extraction and Semiquantitative Reverse}

Transcriptase Polymerase Chain Reaction (RT-PCR)

Monolayer cell cultures were harvested by scraping. The total RNA was extracted using a reagent (TRIzol; Invitrogen Life Technologies) and subjected to RT-PCR amplification of osteopontin and bone morphogenetic protein 2 (BMP2), according to the conditions previously described by Chen et al. (22). Primer sets and their respective annealing temperatures used in this study were as follows: BMP2-forward primer 5'-GGTCCTTGCACCAAGATGAAC-3'; reverse primer 5'-CAACCCTCCACAACCATGTC-3'; temperature, $62^{\circ} \mathrm{C}$; osteopontin-forward primer 5'-ATGAGATTGGCAGTGATT-3'; reverse primer 5'-GTTGACCTCAGAAGATGA-3'; temperature, $48.8^{\circ} \mathrm{C}$. Glyceraldehyde 3-phosphate dehydrogenase was used as an internal control (forward primer 5' - GCTCTCCAGAACATCATCCCTGCC-3'; reverse primer 5'-CGTTGTCATACCAGGAAATGAGCTT-3'; temperature, $\left.55^{\circ} \mathrm{C}\right)$. PCR products were then separated on a $1 \%$ agarose gel (Agarose I; AMRESCO) and visualized with ethidium bromide staining. Images were analyzed using FloGel-I (Fluorescent Gel Image System).

\section{Bone Mineral Density (BMD) Measurement}

Dual-energy x-ray absorptiometry was used to measure BMD in the spine, knee, and femurs. This test was initially performed at time 0 (ovariectomy was performed at $0.5 \mathrm{mo}$ ) and once a month thereafter over a period of 3 mo for different treatment regimes (the endpoint of the experiment was set at 3 mo after the ovariectomy). The BMD of the spine, knees, and femurs was measured and collected using a densitometer (XR-36; Norland Corp.; host software revision 2.5.3, scanner software revision 2.0.0). Statistical analysis was performed using the log-rank test.

\section{Ultrastructural Analysis}

Bone samples from all groups were collected and processed using scanning electron microscopy (SEM). The distal part of the femur and the vertebral body of the third lumbar vertebra were trimmed in the sagittal plane and treated with $30 \%$ potassium hydroxide $(\mathrm{KOH})$ to expose trabecular bones. Bone samples were then dehydrated in acetone and freeze-dried, subsequently mounted on stubs, and coated with gold or palladium using an ion sputter. The processed samples were examined with a microscope (S-3500 N; Hitachi).

\section{Immunohistochemistry and Western Blot Analysis}

Bone sections (10 $\mu \mathrm{m}$ thick) from the lumbar spine, femur, and tibia of the recipient mice were collected, washed, and fixed using $10 \%$ paraformaldehyde solution in phosphate-buffered saline at room temperature. All primary antibodies used were purchased from Santa Cruz Biotechnologies Inc. (BMP2 [sc-6895], osteopontin [sc-10593], and GFP [sc-5385], 1:200 dilution). Respective secondary antibodies were used (1:2,000 dilution) and immunoperoxidase reactions were performed using a Vectastain Universal Elite ABC Kit (Vector Laboratories) according to the manufacturer's protocol. Immunoblotting of control and PRP-treated NIH3T3-G protein extracts was performed according to a standard protocol. Briefly, harvested NIH3T3-G cells were lysed with radioimmunoprecipitation assay $(25 \mathrm{mM}$ Tris- $\mathrm{HCl}, \mathrm{pH} 7.6 ; 150$ $\mathrm{mM} \mathrm{NaCl} ; 1 \% \mathrm{NP}-40 ; 1 \%$ sodium deoxycholate; and $0.1 \%$ sodium dodecylsulfonate [SDS]) and extraction buffers (Pierce). Cell lysates were separated by SDS-polyacrylamide gel electrophoresis. Equal amounts of both control and PRP-treated samples were loaded. For Western blotting, proteins from gels were transferred to the polyvinylidene difluoride membrane (pore size, $0.5 \mu \mathrm{m}$; Schleicher and Schuellx) using a Mini Trans-Blot Cell apparatus (Bio-Rad Laboratories). Immunodetection reaction was visualized by SuperSignal West Pico Chemiluminescent substrate (Biolynx Inc.).

\section{RESULTS}

\section{PRP Induces Osteoblastic Differentiation of NIH3T3-G Cells}

To induce proliferation and osteoblastic differentiation of NIH3T3-G cells in vitro, NIH3T3-G cells were cultured in PRP medium. PRP medium was calibrated on the basis of a 
TGF $\beta 1$ concentration of $750 \mathrm{pg} / \mathrm{mL}$. Control conditions involved culturing NIH3T3-G cells in $1 \%$ fetal CS. The NIH3T3-G cells treated with PRP demonstrated higher proliferative activity than did the group treated with $1 \%$ fetal CS (Fig. 1A). Incubation of NIH3T3-G cells in PRP medium induced the expression of osteoblast-specific markers, osteopontin and BMP2 (Fig. 1B) and ALP (Fig. 1C). In Figure 1C, without PRP treatment, minimal ALP staining was observed in a coculture of NIH3T3-G and BMCs (mimicking the physiologic microenvironment), in BMCs cultured alone, and in NIH3T3-G cultured alone. When PRP was added to the culture medium, the overall cytoplasmic ALP staining was significantly increased in all cell culture groups. PRP/NIH3T3-G cells demonstrated a higher degree of cytoplasmic ALP staining than did both the PRP/NIH3T3-G/BMC and the BMC-alone groups. In addition, the PRP/NIH3T3-G cells exhibited more prominent bone nodule formation at the end of a 21-d culture period than did the NIH3T3-G cells without PRP treatment, as demonstrated by von Kossa staining (Fig. 1D). Furthermore, the osteoinductive effect of PRP was estimated and expressed as the ratio of osteopontin-positive cells or total number of cells. It is estimated that PRP/NIH3T3-G cells had an 8-fold increase in osteopontin expression (Supplemental Fig. 1; supplemental materials are available online only at http://jnm.snmjournals.org).

\section{Transplantation of PRP/NIH3T3-G Cells Effectively Restores BMD in OVX-SAMP8 Mice}

SAMP8 mice, compared with the SAMR1 mice (23) (which served as the control group), exhibited a pronounced acceleration of the aging process at 4 mo of age. Ovariectomy was performed at this time to induce severe osteoporosis in these animals (OVX-SAMP8). Starting 2 wk after the ovariectomy, the BMD of the spine, knee joints (left/right), and femurs (left/right) of these animals was monitored over a period of $3.5 \mathrm{mo}$. As demonstrated in Figure 2, transplantation of PRP/NIH3T3-G cells significantly improved BMD of OVX-SAMP8 mice, compared with vehicle-treated OVX-SAMP8 mice, 4 mo after treatment. PRP or NIH3T3-G alone did not exhibit the same extent of osteogenic effect in OVX-SAMP8 mice as they did in PRP/NIH3T3-G-treated mice (Supplemental Fig. 2). At this time, BMD scores in PRP/NIH3T3-G-treated OVXSAMP8 mice were comparable to those of the control SAMR1 animals, indicating that PRP/NIH3T3-G cell transplantation induced bone regeneration and reversed bone mass loss in these osteoporotic mice.

\section{In Vivo Imaging of Transplanted PRP/NIH3T3-G Cells}

Fluorescent imaging was used for tracking the transplanted PRP/NIH3T3-G cells. In vivo fluorescent images of hind limbs from PRP/NIH3T3-G-treated animals demonstrated the presence of transplanted PRP/NIH3T3-G in the right femur (inferior to the transplantation site) $8 \mathrm{~d}$ after transplantation (Fig. 3A, left panel) and in the contralateral hind limb after $23 \mathrm{~d}$ (Fig. 3A, right panel), suggesting that the transplanted PRP/NIH3T3-G cells were able to survive and proliferate in the bone marrow of the recipient animals for at least $3 \mathrm{wk}$. The flowcytometric analysis on total bone marrow cells obtained from PRP/NIH3T3-G-treated OVXSAMP8 mice 1 mo after transplantation demonstrated a population of cells with strong green fluorescence (Fig. 3B).

Immunohistochemical analysis of the bone sections obtained $15 \mathrm{~d}, 1 \mathrm{mo}$, and 4 mo after transplantation (used in addition to fluorescent imaging to track the transplanted
FIGURE 1. In vitro proliferation and osteoblastic differentiation of PRP/ NIH3T3-G cells. (A) Comparative proliferation profiles of NIH3T3-G cells in PRP-enriched medium and CS were demonstrated by MTT assay (optical densities, mean $\pm S D$ for 3 separate replicates) over a period of $9 \mathrm{~d}$. (B) Cytoplasmic ALP staining during osteogenic differentiation of PRP/NIH3T3-G. Upper and lower rows represent coculture of NIH3T3-G and BMCs, culture of BMCs alone, and culture of NIH3T3-G cells alone in PRP-free (top row) or PRP-enriched (bottom row) medium for $7 \mathrm{~d}$, respectively. (C) Upregulation of messenger RNA (left panels) and protein (right panels) expression levels of osteopontin and BMP2 in NIH3T3-G cells without $(-)$ or with $(+)$ incubation in PRP-enriched medium. Densitometrically quantified data are presented in lower insets; $\beta$-actin levels served as internal control. (D) Increase in number of bone nodules (white arrows) detected by von Kossa staining was in PRP/NIH3T3-G cells, compared with NIH3T3-G cells without PRP treatment. GAPDH = glyceraldehyde-3-phosphate dehydrogenase; OD = optical density; OPN $=$ osteopontin. $t$ test, ${ }^{\star} P<0.05$.

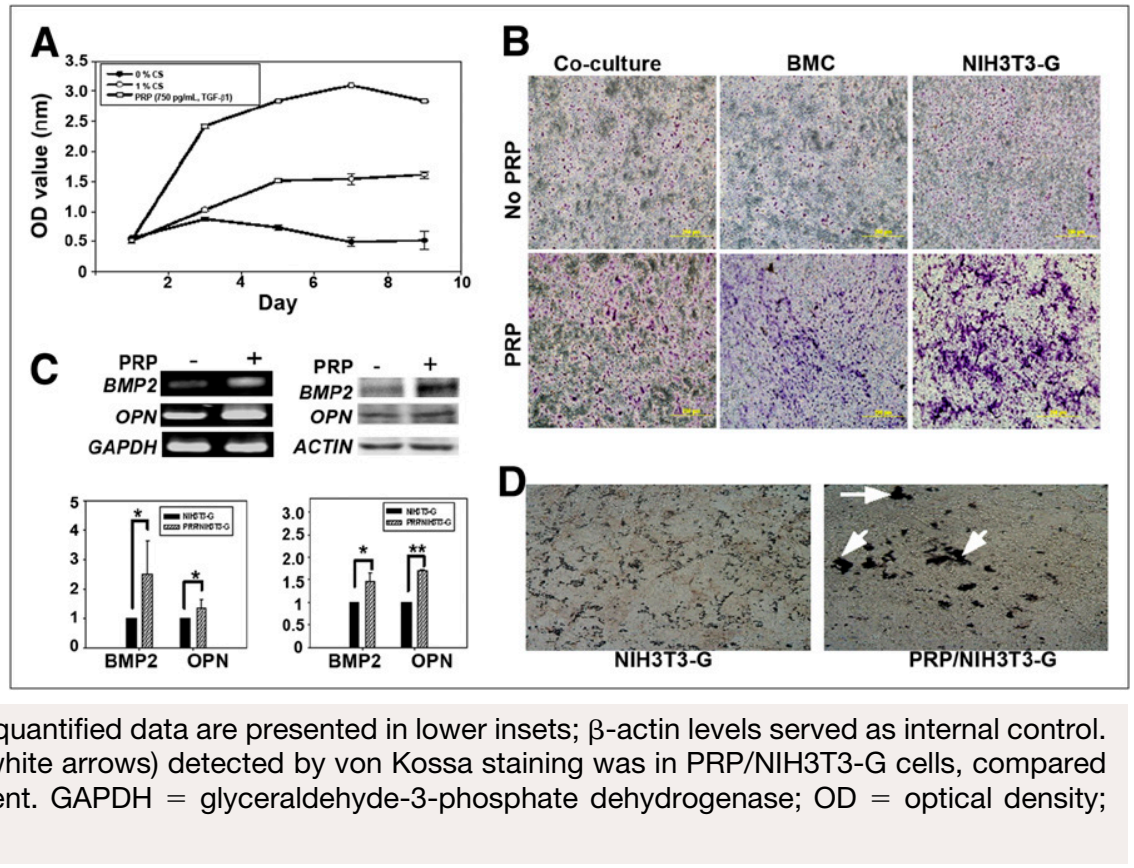




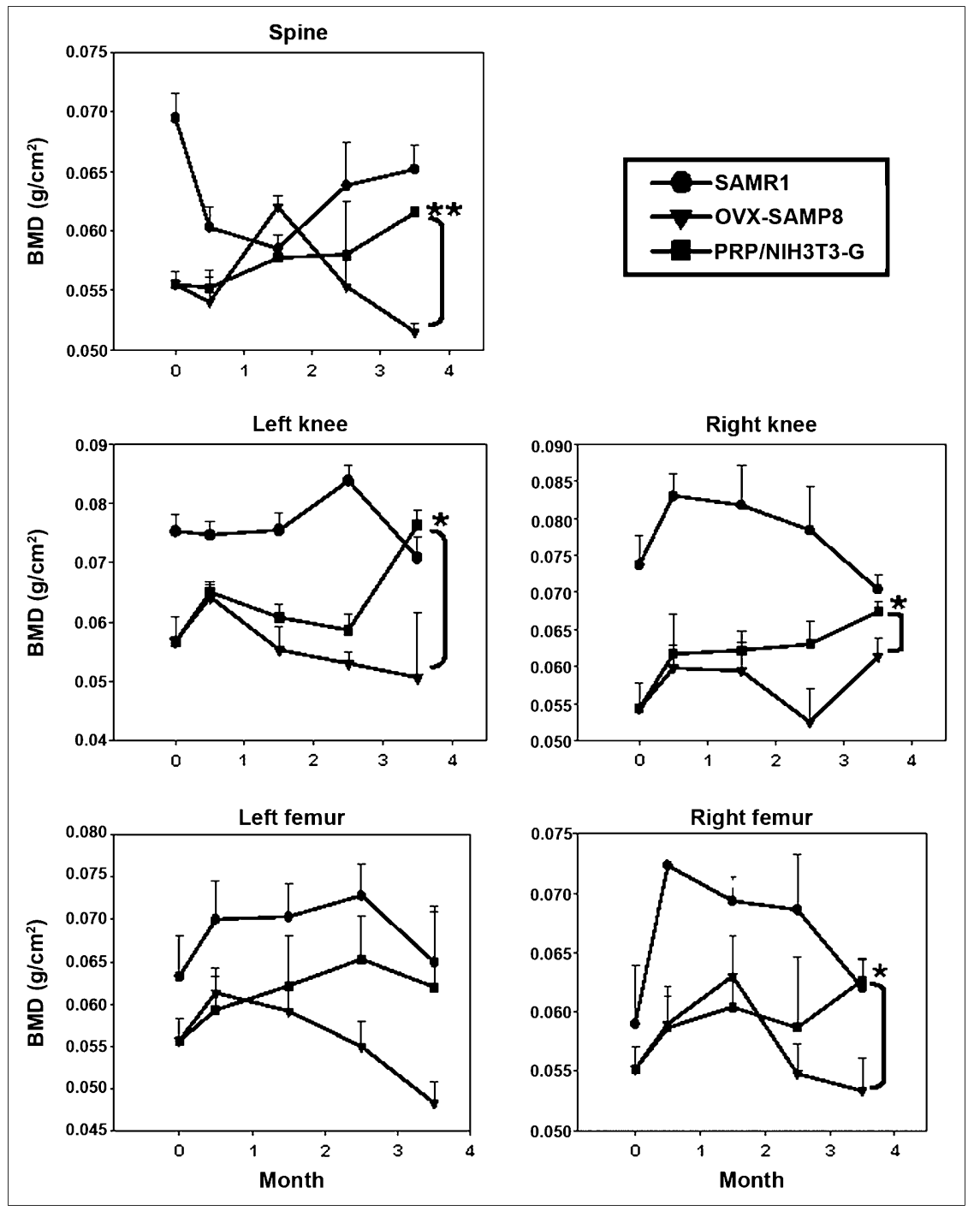

FIGURE 2. Quantitative analysis of BMD in PRP/NIH3T3-G-treated OVXSAMP8 mice and control mice. BMD scores from various parts of skeleton in OVX-SAMP8 mice were plotted over experimental period of 3.5 mo according to different treatment regimes. $t$ test, ${ }^{\star} P<0.05$.

cells) revealed the migration of the PRP/NIH3T3-G cells. GFP-positive (Fig. 4A, arrows) cells were detected in the right knee (transplantation site) and femur, indicating the survival of the transplant and migration to the femur 0.5 mo after transplantation (Fig. 4A, left panels). Meanwhile, at 1 and 4 mo after transplantation, the number of GFP-expressing cells in the right knee and femur gradually increased. Furthermore, PRP/NIH3T3-G cells were detected in the left knee and femur after 1 mo (Fig. 4A, middle panels) and in the spine after 4 mo (Fig. 4A, right panels), demonstrating the migratory ability of these cells.

\section{Ultrastructural Analysis-SEM}

Bone sections from the spine and both knees of PRP/ NIH3T3-G-treated and control OVX-SAMP8 mice were compared using SEM after 4 mo of treatment. The spine and right and left knee sections in the PRP/NIH3T3G-treated group (Fig. 4B, right panels), compared with the control animals (Fig. 4B, left panels from top to bottom, respectively), demonstrated increased trabeculation of the bone structure, corroborating higher BMD scores. Evidently, PRP/NIH3T3-G cells not only repopulated and repaired the bone trabecular architecture in the initial transplantation site but also improved bone morphology of the contralateral hind limb.

\section{Promotion of Osteogenesis in OVX-SAMP8 Mice by PRP/NIH3T3-G Treatment}

To investigate whether PRP/NIH3T3-G cells induced osteogenesis in treated OVX-SAMP8 mice, bone sections from the right knee (transplantation site) at 1 mo after transplantation were analyzed for the colocalization of GFP and osteoblast marker osteopontin. Positive GFP signals were detected in the bone at the engraftment site (Fig. 5A). GFP-positive cells were found in the newly formed bone trabecules (Fig. 5B). In addition, both osteopontin and GFP signals colocalized in the same cells, indicating that the 

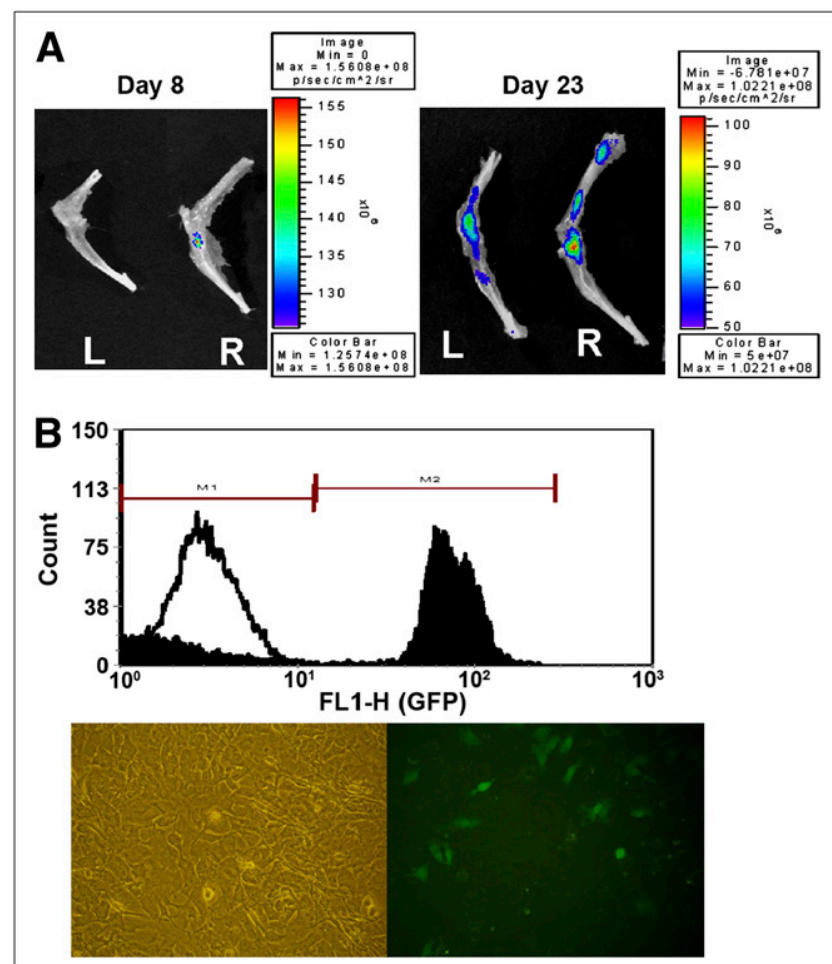

FIGURE 3. In vivo imaging of engrafted PRP/NIH3T3-G cells. (A) In vivo fluorescence images of resected hind limbs from mice at 8 and $23 \mathrm{~d}$ after transplantation, respectively, showed migration of transplanted cells. (B) Isolation of transplanted NIH3T3-G cells from bone marrow of PRP/ NIH3T3-G-treated OVX-SAMP8 mice 1 mo after transplantation. Total bone marrow cells were collected from bone marrow of PRP/NIH3T3-G-treated OVX-SAMP8 mice 1 mo after transplantation, and NIH3T3-G cells were sorted out among other cell types by flow cytometry. (Top) NIH3T3-G cells (M2) from total BMCs, compared with other cells (M1), exhibited strong green fluorescence intensity. (Bottom) NIH3T3-G cells isolated from bone marrow of PRP/ NIH3T3-G-treated OVX-SAMP8 were (left) in vivo expanded and exhibited strong green fluorescence (right) under fluorescence microscope.

PRP/NIH3T3-G cells had maintained their osteoblastic differentiation (Fig. 5B, upper and lower rows).

\section{Extension of Life Span of OVX-SAMP8 Mice by PRP/ NIH3T3-G Transplantation}

In addition to markedly improving bone trabecular architecture and BMD, the PRP/NIH3T3-G cell transplantation extended the life span of OVX-SAMP8 animals, which was significantly longer than that in the untreated OVX-SAMP8 mice and comparable to that in the control SAMPR1 mice (Fig. 6). All untreated OVX-SAMP8 animals died around $11 \mathrm{mo}$; approximately $80 \%$ of both SAMR1 and PRP/NIH3T3-G-treated groups lived up to $16.6 \mathrm{mo}$, a life expectancy similar to the previously reported life expectancy for SAMR1 (12). Intriguingly,
PRP/NIH3T3-G-treated mice outlived both the SAMP8 and the OVX-SAMP8 groups (Fig. 6).

\section{DISCUSSION}

In this study, using optical imaging we demonstrated the efficacy of a simple and yet unique approach for severe senile osteoporosis using intraosseous transplantation of fibroblasts in combination with a growth factor mixture, PRP. We have established an ideal animal model to study osteoporosis: the OVX-SAMP8 animals with low BMD scores and same-aged controls, SAMR1 animals with normal BMD. The OVX-SAMP8 model of severe postmenopausal osteoporosis has clinical manifestations of osteoporosis similar to those in human patients because the model mimics both age and postmenopausal ovarian involution-induced changes in bone physiology, architecture, and mineral density. We chose NIH3T3 fibroblasts because these cells are readily available and are well characterized. In fact, genetically engineered dermal fibroblasts have been used as an alternative cell source for bone regeneration in animals $(10,11)$ because of the ease of harvest from autologous donors and high capacity for in vitro expansion and genetic manipulations. In addition, NIH3T3 is a clonal fibroblastic cell line from a mouse embryo (24); Shui and Scutt (15) demonstrated that on $1 \alpha-$ 25-dihydroxyvitamin D3 and dexamethasone treatment, these cells acquired an osteoblastlike phenotype. In the current study, we have verified the ability of NIH3T3-G cells to differentiate into osteoblastlike cells in vitro when incubated in medium enriched with PRP. Supported by previous findings, PRP represents a useful growth factor cocktail containing TGF- $\beta 1$, TGF- $\beta 2$, vascular endothelial growth factor, platelet-derived growth factor (PDGF), and insulinlike growth factor, all of which are naturally released from platelets and are essential in mesenchymal stem cell differentiation including osteoprogenic cells $(17,20,21,25)$. In addition, PDGF and TGF- $\beta$ in PRP have been shown to stimulate cell migration and inhibit cell proliferation or differentiation, respectively (25). Osteoblastlike differentiation of PRP-treated NIH3T3-G cells manifested in an increased expression of osteogenic makers, such as BMP2 and osteopontin, at both messenger RNA and protein levels, increased cytoplasmic ALP expression, and increased formation of bone nodules in vitro. Our PRP/NIH3T3-G system, in which no genetic manipulation was required to achieve fibroblast-osteoblast transformation, poses an inherent advantage over fibroblasts transduced with osteoinductive genes such as RUNX2 (10,26), BMPs (27-29), and LMP-3 (11). In addition, the copy number of viralmediated transfused osteoinductive genes could not be controlled in these genetically engineered fibroblasts. Thus, predifferentiation of NIH3T3-G cells by PRP provides not only a physiologic but also a clinically oriented patientindividualized method for obtaining osteoinductive cells for cell-based therapy of osteoporosis. 


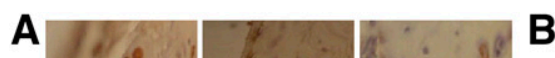

B Control PRPINH3T3-0
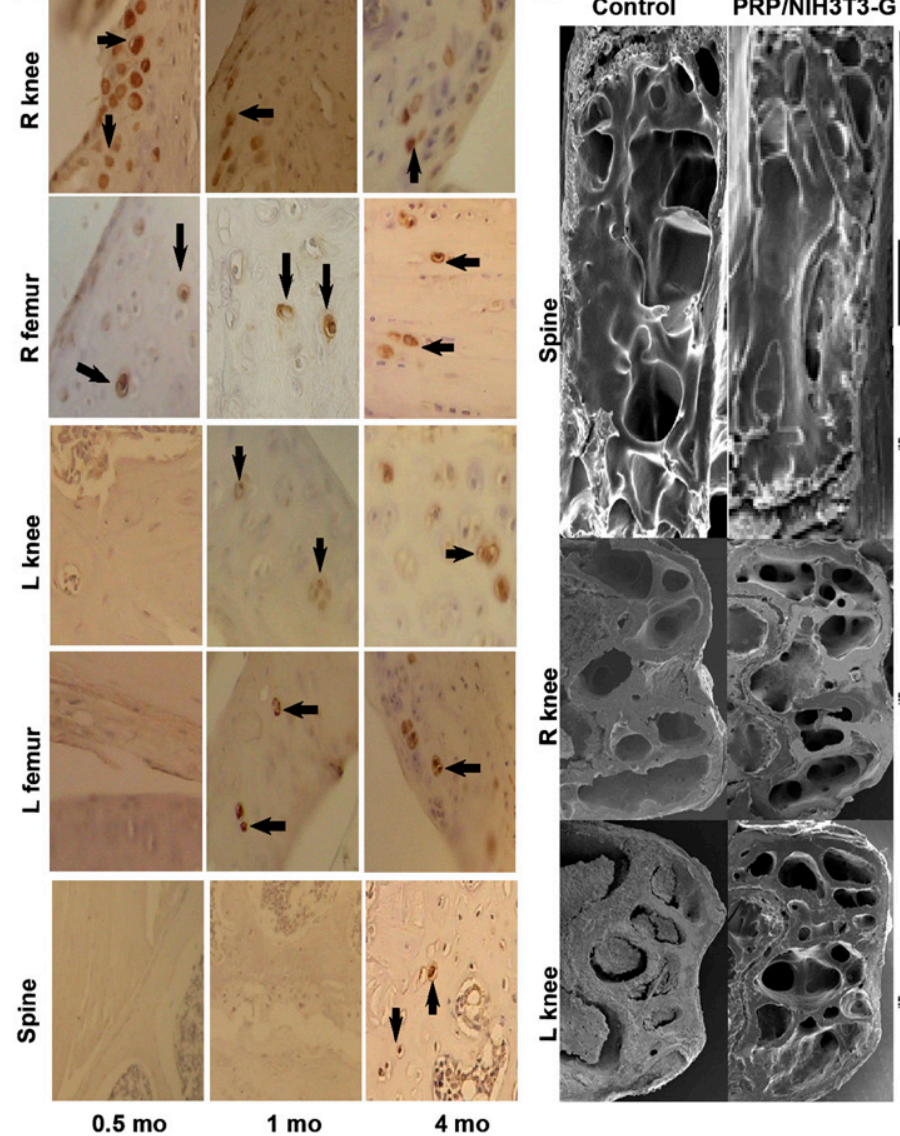

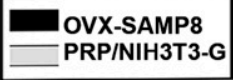

SEM: Spine

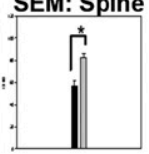

SEM: R knee

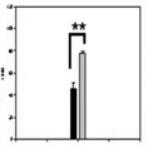

SEM: L knee

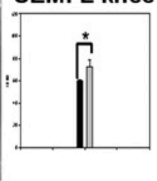

FIGURE 4. Bone morphologic characterizations of PRP/NIH3T3-Gtransplanted OVX-SAMP8 mice. (A) GFP-positive cells were detected in various parts of skeleton at indicated times. At day 15, GFP-positive signals (brown signals indicated by arrows) were observed only in right knee and femur; GFP-positive signals in left knee and femur were observed at $1 \mathrm{mo}$ and then in spine at $4 \mathrm{mo}$. (B) Comparative scanning electron micrographs of bone sections from both control and treated groups after 4 mo. Respective inserts demonstrate ratio of BMD scores obtained in areas of trabeculae and compact bones over total observed bone areas. $t$ test, ${ }^{\star} P<0.05,{ }^{\star \star} P<0.01$.
The capacity of PRP/NIH3T3-G cells for active migration across different skeletal bones was clearly demonstrated in the current study by optical imaging. Transplantation of PRP/NIH3T3-G cells into 1 of the bone marrow cavities (in the right femur) of OVX-SMP8 mice resulted in generalized skeletal reversal of osteoporosis, which was due to migration of these cells from the original site of implantation into the distal bones. This observation was corroborated by in situ analysis of GFP fluorescence imaging in osteoblastlike cells (expressing osteopontin) both at the site of implantation and into the distal bones (i.e., spine and contralateral femur). The tropism of these cells into the osteoporotic sites could be mediated by general inflammatory stimuli, such as those in the SDF1-CXCR4 axis (data not shown), and specific growth factors and cytokines (e.g., TGF $\beta$, PDGF, and BMP) (30). As demonstrated in the current study, expression of BMP2 and osteopontin in NIH3T3-G cells significantly increased after PRP-induced osteoblastic differentiation. The upregulation of BMP2 and osteopontin favors the bone-formation process over the absorption process, thereby ameliorating osteoporosis.

The combined treatment of PRP/NIH3T3-G significantly improved the BMD scores and overall skeletal bone architecture in OVX-SAMP8 animals, compared with those seen in the control SAMR1 group. As we demonstrated by optical imaging, the PRP/NIH3T3-G cells had migrated toward and engrafted into the progressively developing osteoporotic lesions in OVX-SAMP8 mice. The osteoblastlike activity of these cells after engraftment into the osteoporotic bones resulted in the gradual reorganization and normalization of bone morphology, as manifested by a significantly improved trabecular architecture of the bone populated with the PRP/NIH3T3-G cells and higher overall BMD scores in the treated animals.

Results from the present study are in accord with a previous report on successful treatment of ovariectomyinduced osteoporosis in a rabbit model using transplantation of GFP-expressing autologous mesenchymal stem cells (31). However, the ovariectomized rabbit model is more suitable for studies of osteoporosis induced by ovarian dysfunction. In contrast to the OVX-SAMP8 model with accelerated aging, this rabbit model did not address the factor of aging in the development of senile osteoporosis. Thus, the OVX-SAMP8 model and the results of our current study have more biologic relevance to both the establishment of a working model and the development of novel therapies for postmenopausal and senile osteoporosis in general.

The OVX-SAMP8 mice used in our study differ from the previously described SAMP6 animal model $(32,33)$. In the SAMP6 study, transplantation of allogeneic bone marrow cells from normal mouse strains into osteoporotic SAMP6 


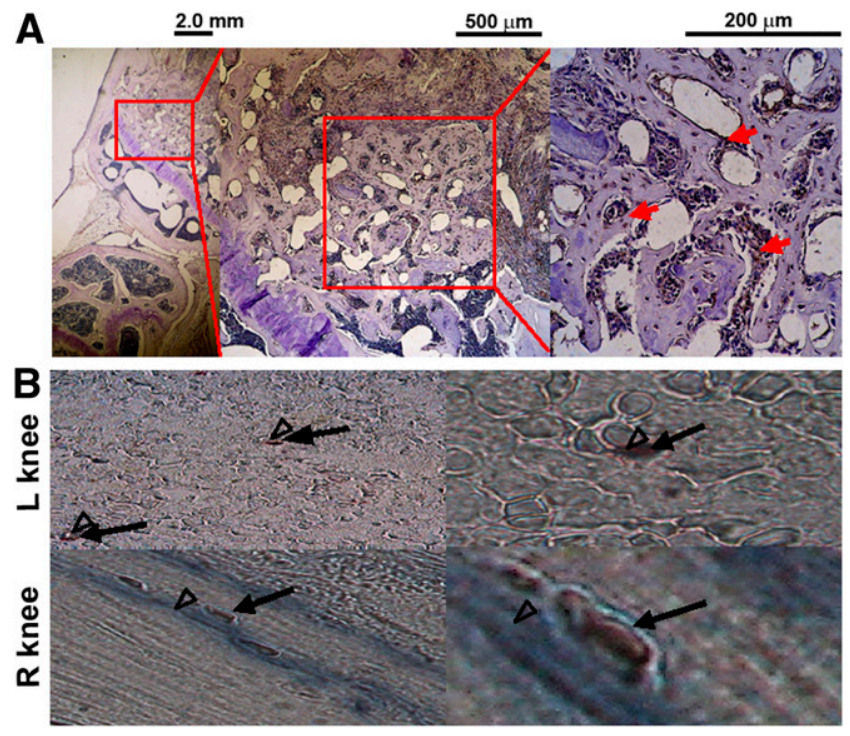

FIGURE 5. Immunohistochemical characterization of osteoblastlike differentiation of GFP-containing cells in OVXSAMP8 mice. (A) Numerous GFP-positive cells were detected at 1 mo in new bone (GFP, brown). (B) Colocalization of staining for osteoblastic marker OPN (stained blue, arrowheads) in GFP-positive cells (stained red, arrows) in both knees of PRP/NIH3T3-G-treated OVX-SAMP8 mice. Upper and lower left panels, magnification $=200 \times$; upper and lower right panels, magnification $=1,000 \times$ ).

mice restored the bone architecture (34). However, the latter study did not address the hormonal changes that contribute to the development of severe postmenopausal senile osteoporosis. In contrast, the OVX-SAMP8 mice exhibited a pronounced decrease in BMD due to both

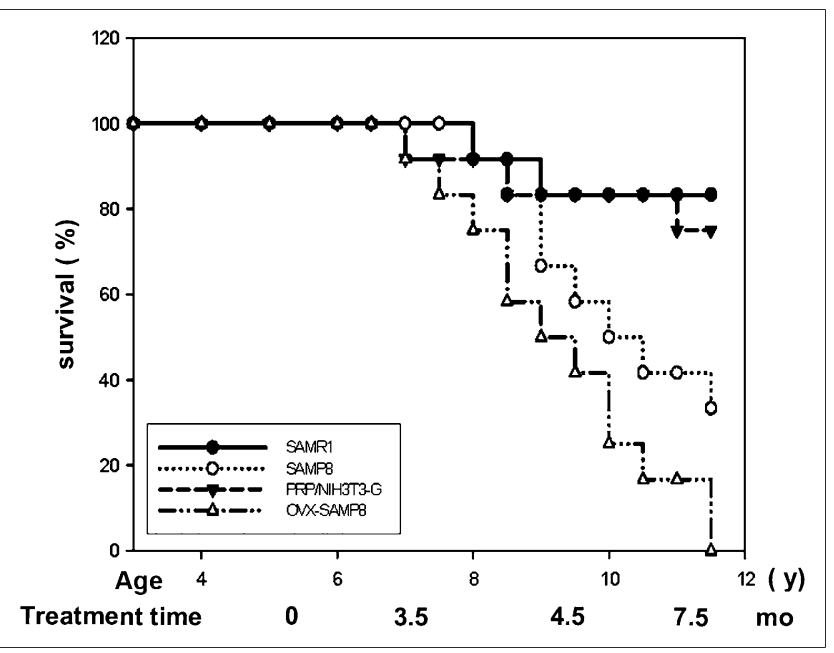

FIGURE 6. Survival of OVX-SAMP8 mice treated with PRP/NIH3T3-G cells. Kaplan-Meyer survival plots for different groups of mice ( $n=12$ in each group) with and without transplantation of the PRP/NIH3T3-G cells: control SAMR1 mice, SAMP8 mice, OVX-SAMP8 mice treated with PRP/ NIH3T3-G cells, and untreated OVX-SAMP8 mice. senescence and the loss of estrogen after ovariectomy. Thus, we have answered the question of whether the transplanted normal osteoprogenic cells could engraft into the osteoporotic bone and reconstitute their osteoblastic functions in the absence of estrogen. Collectively, our findings and those of Wang et al. (31) and Takada et al. (34) indicate that not only the hemopoietic system but also the bone microenvironment, trabecular architecture, and BMD could potentially be normalized by progenitor cell transplantation into postmenopausal geriatric individuals, resulting in an amelioration of the imbalance between bone absorption and formation. Up to date, several drugs for the prevention and treatment of osteoporosis have been approved by the Food and Drug Administration. However, these drugs (or compounds, all anticatabolic), including bisphosphonates, calcitonin, and selective estrogen receptor modulators, work by inhibiting bone resorption and only prevent further loss of bone. These agents do not stimulate new bone formation. The only Food and Drug Administration-approved compound capable of stimulating neobone formation (and thus reversing bone loss) is parathyroid hormone (PTH). However, PTH must be administered subcutaneously and presents potential side effects such as treatment-associated hypercalcemia and hypercalciuria. In addition, the duration of treatment with PTH is limited to 18 mo in Europe and 24 mo in the United States, because rodent studies demonstrated that high doses of PTH promoted the development of osteosarcomas (35). Thus, we believe that our system could offer a better alternative for a new therapeutic strategy for osteoporosis.

Furthermore, our current studies demonstrate that therapy with PRP/NIH3T3-G appeared to unexpectedly prolong the life span of the OVX-SAMP8 animals (Fig. 6). This phenomenon raises an important question regarding the mechanism involved in life-span prolongation of PRP/ NIH3T3-G-treated animals. We hypothesize that PRP/ NIH3T3-G treatment not only prevented osteoporosis in these mice but also improved other senescence-related conditions by engraftment into the degenerative lesions of other rapidly aging tissues and organs and potentially improving (regenerating) their structure and function. Additional studies are required to explore this hypothesis. Nevertheless, in view of our results, stem cell transplantation in combination with PRP/NIH3T3-G treatment could potentially be applicable for treatment of HutchinsonGilford progeria syndrome, which is a rare, genetically predetermined condition characterized by accelerated aging in children (36). Our results also provide support for the concept of rejuvenation therapy using stem cell minitransplants for the prolongation of life (37).

\section{CONCLUSION}

We have developed a potentially promising progenitor cell-based therapy of severe senile osteoporosis, in which PRP could provide appropriate growth factors for predif- 
ferentiation of osteoprogenic cells (i.e., derived from either bone marrow or cord blood) into osteoblastlike cells for transplantation. This novel therapeutic platform could be applied to the treatment of osteoporosis and other skeletalrelated disorders in human patients and to the prolongation of life expectancy in general.

\section{ACKNOWLEDGMENTS}

We thank Wen-Tien Hsiao and Milligrams Instruments Co., Ltd., for their excellent technical assistance in BMD measurement and Ching-Yu Tsai for her surgical assistance with the ovariectomies. This research was supported by the following grants and agencies: Department of Health (DOH), DOH96-TD-G-111-013; National Science Council, NSC 97-2314-B-038-0330MY3; Core Facility grant 973112-B-010-016; and Stem Cell Research Center and Cancer Center, Taipei Medical University Hospital, Taipei, Taiwan.

\section{REFERENCES}

1. Raisz LG. Pathogenesis of osteoporosis: concepts, conflicts, and prospects. J Clin Invest. 2005;115:3318-3325.

2. Reginster JY, Burlet N. Osteoporosis: a still increasing prevalence. Bone. 2006;38(2, suppl 1):S4-S9.

3. Rosen CJ. Clinical practice: postmenopausal osteoporosis. N Engl J Med. 2005; 353:595-603.

4. Chapurlat RD, Delmas PD. Drug insight: bisphosphonates for postmenopausal osteoporosis. Nat Clin Pract Endocrinol Metab. 2006;2:211-219.

5. Whitfield JF. Osteoporosis-treating parathyroid hormone peptides: what are they? what do they do? how might they do it? Curr Opin Investig Drugs. 2006; 7:349-359.

6. Cao JJ, Wronski TJ, Iwaniec U, et al. Aging increases stromal/osteoblastic cellinduced osteoclastogenesis and alters the osteoclast precursor pool in the mouse. J Bone Miner Res. 2005;20:1659-1668.

7. Chen TL. Inhibition of growth and differentiation of osteoprogenitors in mouse bone marrow stromal cell cultures by increased donor age and glucocorticoid treatment. Bone. 2004;35:83-95.

8. Kawaguchi H. Molecular backgrounds of age-related osteoporosis from mouse genetics approaches. Rev Endocr Metab Disord. 2006;7:17-22.

9. Labrie JE III, Borghesi L, Gerstein RM. Bone marrow microenvironmental changes in aged mice compromise $\mathrm{V}(\mathrm{D}) \mathrm{J}$ recombinase activity and B cell generation. Semin Immunol. 2005;17:347-355.

10. Phillips JE, Guldberg RE, Garcia AJ. Dermal fibroblasts genetically modified to express Runx $2 / \mathrm{Cbfa} 1$ as a mineralizing cell source for bone tissue engineering. Tissue Eng. 2007;13:2029-2040.

11. Lattanzi W, Parrilla C, Fetoni A, et al. Ex vivo-transduced autologous skin fibroblasts expressing human Lim mineralization protein-3 efficiently form new bone in animal models. Gene Ther. 2008;15:1330-1343.

12. Takeda T, Matsushita T, Kurozumi M, Takemura K, Higuchi K, Hosokawa M. Pathobiology of the senescence-accelerated mouse (SAM). Exp Gerontol. 1997;32:117-127.

13. Flood JF, Morley PM, Morley JE. Age-related changes in learning, memory, and lipofuscin as a function of the percentage of SAMP8 genes. Physiol Behav. 1995;58:819-822.

14. Morley JE, Kumar VB, Bernardo AE, et al. Beta-amyloid precursor polypeptide in SAMP8 mice affects learning and memory. Peptides. 2000;21:1761-1767.

15. Shui C, Scutt AM. Mouse embryo-derived NIH3T3 fibroblasts adopt an osteoblast-like phenotype when treated with 1 1 ,25-dihydroxyvitamin $\mathrm{D}_{3}$ and dexamethasone in vitro. J Cell Physiol. 2002;193:164-172.
16. Li G, Peng H, Corsi K, Usas A, Olshanski A, Huard J. Differential effect of BMP4 on NIH/3T3 and $\mathrm{C} 2 \mathrm{C} 12$ cells: implications for endochondral bone formation. J Bone Miner Res. 2005;20:1611-1623.

17. Chen WH, Lo WC, Lee JJ, et al. Tissue-engineered intervertebral disc and chondrogenesis using human nucleus pulposus regulated through TGF- $\beta 1$ in platelet-rich plasma. J Cell Physiol. 2006;209:744-754.

18. Liu Y, Kalen A, Risto O, Wahlstrom O. Fibroblast proliferation due to exposure to a platelet concentrate in vitro is $\mathrm{pH}$ dependent. Wound Repair Regen. 2002;10:336-340.

19. Arpornmaeklong P, Kochel M, Depprich R, Kubler NR, Wurzler KK. Influence of platelet-rich plasma (PRP) on osteogenic differentiation of rat bone marrow stromal cells: an in vitro study. Int J Oral Maxillofac Surg. 2004;33:60-70.

20. Tozum TF, Demiralp B. Platelet-rich plasma: a promising innovation in dentistry [abstract]. J Can Dent Assoc. 2003;69:664.

21. Kanno T, Takahashi T, Tsujisawa T, Ariyoshi W, Nishihara T. Platelet-rich plasma enhances human osteoblast-like cell proliferation and differentiation. J Oral Maxillofac Surg. 2005;63:362-369.

22. Wan DC, Shi YY, Nacamuli RP, Quarto N, Lyons KM, Longaker MT. Osteogenic differentiation of mouse adipose-derived adult stromal cells requires retinoic acid and bone morphogenetic protein receptor type IB signaling. Proc Natl Acad Sci USA. 2006;103:12335-12340.

23. Takeda T, Hosokawa M, Takeshita S, et al. A new murine model of accelerated senescence. Mech Ageing Dev. 1981;17:183-194.

24. Jainchill JL, Aaronson SA, Todaro GJ. Murine sarcoma and leukemia viruses: assay using clonal lines of contact-inhibited mouse cells. J Virol. 1969;4: 549-553.

25. Celotti F, Colciago A, Negri-Cesi P, Pravettoni A, Zaninetti R, Sacchi MC. Effect of platelet-rich plasma on migration and proliferation of SaOS-2 osteoblasts: role of platelet-derived growth factor and transforming growth factor-beta. Wound Repair Regen. 2006;14:195-202.

26. Phillips JE, Hutmacher DW, Guldberg RE, Garcia AJ. Mineralization capacity of Runx2/Cbfa1-genetically engineered fibroblasts is scaffold dependent. Biomaterials. 2006;27:5535-5545.

27. Rutherford RB, Moalli M, Franceschi RT, Wang D, Gu K, Krebsbach PH. Bone morphogenetic protein-transduced human fibroblasts convert to osteoblasts and form bone in vivo. Tissue Eng. 2002;8:441-452.

28. Hirata K, Tsukazaki T, Kadowaki A, et al. Transplantation of skin fibroblasts expressing BMP-2 promotes bone repair more effectively than those expressing Runx2. Bone. 2003;32:502-512.

29. Krebsbach PH, Gu K, Franceschi RT, Rutherford RB. Gene therapy-directed osteogenesis: BMP-7-transduced human fibroblasts form bone in vivo. Hum Gene Ther. 2000;11:1201-1210.

30. Zhu W, Boachie-Adjei O, Rawlins BA, et al. A novel regulatory role for stromalderived factor-1 signaling in bone morphogenic protein- 2 osteogenic differentiation of mesenchymal C2C12 cells. J Biol Chem. 2007;282:18676-18685.

31. Wang Z, Goh J, Das DS, et al. Efficacy of bone marrow-derived stem cells in strengthening osteoporotic bone in a rabbit model. Tissue Eng. 2006;12:17531761 .

32. Ishihara A, Roy RR, Ohira Y, et al. Effects of aging and exercise on density and cross-sectional area of femur in senescence-accelerated mouse prone 6 . J Musculoskelet Neuronal Interact. 2003;3:162-169.

33. Chen H, Yao XF, Emura S, Shoumura S. Morphological changes of skeletal muscle, tendon and periosteum in the senescence-accelerated mouse (SAMP6): a murine model for senile osteoporosis. Tissue Cell. 2006;38:325-335.

34. Takada K, Inaba M, Ichioka N, et al. Treatment of senile osteoporosis in SAMP6 mice by intra-bone marrow injection of allogeneic bone marrow cells. Stem Cells. 2006;24:399-405.

35. Vahle JL, Sato M, Long GG, et al. Skeletal changes in rats given daily subcutaneous injections of recombinant human parathyroid hormone (1-34) for 2 years and relevance to human safety. Toxicol Pathol. 2002;30:312-321.

36. Brune T, Bonne G, Denecke J, et al. Progeria: a new kind of laminopathyreport of the First European Symposium on Progeria and creation of EUROProgeria, a European consortium on progeria and related disorders. Pediatr Endocrinol Rev. 2004;2:39-45.

37. Conboy IM, Conboy MJ, Wagers AJ, Girma ER, Weissman IL, Rando TA. Rejuvenation of aged progenitor cells by exposure to a young systemic environment. Nature. 2005;433:760-764. 